\title{
Understanding the Nebular Spectrum of SN 1987A
}

\author{
By RICHARD M C CR A Y \\ JILA, University of Colorado, Boulder, CO 80309-0440, USA
}

\begin{abstract}
The nebular spectra of supernovae differ from those of better-known emission nebulae in that many of the emission lines are optically thick. Here we sketch the theory for interpreting such spectra, and show how it can be used to interpret prominent emission line systems in the spectrum of SN 1987A. As examples, we describe: (1) a simple method to infer the density of OI from observations of the evolution of the doublet ratio in $[O \mathrm{I}] \lambda \lambda 6300 ;(2)$ new kind of hydrogen recombination line spectrum; (3) an analysis showing that the Ca II infrared emission lines must come from primordial, not newly-synthesized, calcium; (4) a theory for the $\mathrm{Fe} / \mathrm{Co} / \mathrm{Ni}$ emission lines that shows that the inner envelope of SN 1987A must have a foamy texture, in which low density radioactive bubbles of $\mathrm{Fe} / \mathrm{Co} / \mathrm{Ni}$ reside in a massive substrate of hydrogen, helium, and other elements.
\end{abstract}

\section{Introduction}

Conventional wisdom holds that supernova explosions produce most of the heavy elements in the universe, and a major goal of astronomy is to test this hypothesis through observations of supernova spectra. For this purpose, SN 1987A should be a Rosetta Stone. We have observed its spectrum in far greater detail than that of any other supernova: at wavelength bands, such as gamma rays and far infrared, where no other supernova has been observed; with almost daily (nightly!) observations continuing for more than seven years after outburst; and with unprecedented spectral resolution (McCray 1993). What have we learned about the debris of SN 1987A from such observations? About element synthesis? About the physical conditions in the debris? How can we use the knowledge gained from SN 1987A to interpret the spectra of other, more distant supernovae?

We can identify three major components that appear in the spectra of supernovae. The first, called the photospheric spectrum, usually dominates during the first few months after outburst (until maximum, and during the plateau phase of the light curve). The photospheric spectrum is characterized by a strong blackbody-like continuum with a characteristic temperature that levels off at $\sim 5000 \mathrm{~K}$ after a week or two. This continuum is punctuated by strong P-Cygni lines, mainly hydrogen lines in SN II and metal lines in SN I. The second component, called the nebular spectrum, dominates after a few months, when the photospheric recedes to the center of the envelope and the continuum fades. In the nebular phase, most of the luminosity appears as emission lines and it is difficult to discern an optical continuum from the many overlapping weak emission lines. When I use the term nebular spectrum, I mean specifically an emission line spectrum that resembles that of $\mathrm{SN} 1987 \mathrm{~A}$ after four months. It is characterized by lines from neutral and singly-ionized elements and linewidths with FWHM $\sim 3000-4000 \mathrm{~km} \mathrm{~s}^{-1}$; it clearly comes from the glowing radioactive interior of the supernova envelope.

Often, supernova spectra have a third component, which I shall call the interaction spectrum. It is also characterized by strong emission lines, but the lines are very different from those seen in the spectrum of SN 1987A. They typically display emission lines of elements ionized twice or more, having broad, often asymmetric, profiles with FWHM $z 5000 \mathrm{~km} \mathrm{~s}^{-1}$. The interaction spectrum is evidently caused, not by the radioactive 
glow of the inner supernova debris, but by the shocks that result from the impact of the debris with circumstellar matter that has been ejected by the progenitor. Up to now, SN 1987A lacks such a spectrum because it is expanding into a bubble of very low density gas created by its blue-giant progenitor. The interaction spectrum of SN 1987A will only appear $c a$. AD $1999 \pm 3$ when its envelope strikes the circumstellar ring (Suzuki et al 1993; Masai \& Nomoto 1993; Luo, McCray, \& Slavin 1994). But the interaction spectrum is often very prominent, even at relatively early times, in SNe with red giant progenitors, such as SN 1993J (Wheeler \& Filippenko, 1995). With SN 1987A we are lucky, because we can be sure that its nebular spectrum comes only from its radioactive interior and is not contaminated by an interaction spectrum.

Here I give a brief summary of the present state of the art of interpreting the nebular spectrum of SN 1987A. Some of the results came as a surprise to us, but are fairly easy to understand in retrospect. As the reader will see, we have learned less than we might have hoped about element synthesis, but more than we might have hoped about the dynamics and thermodynamics of the envelope. Many of the basic principles are universal, and will undoubtedly be very useful in interpreting the nebular spectra of other supernovae.

Two rather different strategies have been used to model the nebular spectrum of SN 1987A. In the first, one calculates a model for the entire spectrum starting with a model for the supernova envelope characterized by a set of parameters representing element composition, etc. One then adjusts the parameters to achieve a best synthesis of the model with observations. This strategy, represented by the pioneering efforts by Fransson \& Chevalier (1987), Swartz et al (1987), and Colgan \& Hollenbach (1988), had a fair amount of success in predicting and interpreting the evolution of the overall nebular spectrum of SN 1987A; but, as one might expect, did not account for many details.

We have adopted a second strategy, following the pioneering work by Chugai (1987, 1990). Rather than trying to synthesize the whole spectrum at once, we have focused our efforts on developing detailed models for the time development of emission lines from each different element group. We adopted this approach mainly because we suspected (and now we are sure) that the parts of the supernova envelope responsible for emitting the lines of different elements retain their chemical identities - e.g., most of the hydrogen has approximately primordial composition, most of the oxygen is found in zones of relatively pure carbon/oxygen, etc. I.e., hydrodynamic instabilities following the blast caused the supernova envelope to be mixed macroscopically but not microscopically (stirred, not blended). We have attempted to model the emission lines from different element groups, one by one, concentrating on those elements responsible for the brightest lines: hydrogen, oxygen, calcium, and the iron group elements.

Our strategy has advantages and disadvantages. The main disadvantage is that it is painstaking and slow. One can reach an overall gestalt of the emitting region more readily through the first, more synthetic, strategy. The main advantage of our strategy is that, by focusing on one element at a time, we can identify more easily the physical processes that control the line emission, and thereby pin down with greater confidence the physical state of the relevant emitting region. Of course, we hope ultimately to combine the results of our detailed studies to produce a synthetic model for the envelope that fairly represents the development of the entire spectrum. As the reader will see, however, we have a few more obstacles to overcome before we reach that goal.

\section{Emission Line Formation in Supernova Envelopes}

During the nebular phase, most of the luminosity of the supernova envelope emerges as an optical/infrared emission line spectrum. Just as in an H II region or a planetary 
nebula, the emission lines from hydrogen are produced mainly by radiative recombination and those from other elements are produced mainly by electron impact excitation. Thus, one can turn to classic texts such as Osterbrock (1989) for details of the physical processes and diagnostic methods. However, supernova envelopes differ from ordinary nebulae in four important ways:

- Supernova envelopes expand hypersonically (Mach number $\mathcal{M} \sim 10^{3}$ ), so that resonance scattering of a line photon is confined to a region of fractional thickness $\sim \mathcal{M}^{-1}$.

- Supernova envelopes have atomic densities, $n \sim 10^{7}-10^{9} \mathrm{~cm}^{-3}$, that are much higher than the densities, $n \sim 10^{2}-10^{4} \mathrm{~cm}^{-3}$, in typical nebulae. As a result, many emission lines, even the forbidden lines, have high optical depths.

- Supernova envelopes are ionized by gamma rays, which have low ionization crosssections and long mean free paths. As a result, the envelope contains no sharp ionization fronts.

- Because the densities are high and the gamma rays have relatively low flux, the envelope is mostly neutral and much colder than ordinary emission line nebulae.

Thus, if we wish to understand what the nebular spectrum of a supernova is telling us about the physical conditions (densities, temperatures, element composition) of the supernova ejecta, we must modify the standard theory of emission nebulae to account for these differences. Let us address them in turn. See McCray (1993) for more details and references; here we merely summarize the main points and illustrate them with a few simple examples.

\subsection{Envelope expansion and line profiles}

Fortunately, the motion of a supernova envelope can be described by the simplest possible dynamics: Hubble's Law! Because acceleration within the envelope ceases within hours or at most days after the explosion, the distance from the center of any parcel of gas is given by $r=v t$, where $V$ is its radial velocity, and $t$ is the time since the explosion. It follows that the relative recession velocities, $\Delta v=v_{1}-v_{2}$ of any two mass points is related to their separation $\Delta r=r_{1}-r_{2}$ by the law $\Delta v=H_{0} \Delta r$, where the "Hubble constant" $H_{0}=t^{-1}$.

Moreover, the emission lines from a supernova envelope have Doppler widths indicative of expansion velocities of the emitting region $v_{\text {exp }} \sim 1000-3000 \mathrm{~km} \mathrm{~s}^{-1}$, much greater than the thermal velocities $v_{t h} \sim 1-3 \mathrm{~km} \mathrm{~s}^{-1}$ of the line-emitting atoms or moleculest. Thus, the regions of the supernova envelope responsible for emitting different parts of the line profiles are physically separated. In fact, the region responsible for line emission at a given Doppler shift, $\Delta \lambda=\left(v_{\|} / c\right) \lambda_{0}$, is confined to a thin planar section displaced a distance $\Delta z=c t \Delta \lambda / \lambda_{0}$ from the midplane of the supernova envelope, as illustrated in Figure 1. The section has thickness $\delta z \sim\left(v_{t h} / v_{\|}\right) \Delta z$.

It is easy to demonstrate that line emission from a thin spherical shell would have a rectangular line profile. From that fact, one can infer that if the supernova envelope had a laminar, 'onion-like' distribution of element groups (as we believe the progenitor did), the line profiles from different chemical element would have flat tops, with the Doppler widths of the flat tops representing the minimum expansion velocities of the respective elements. But the line profiles in the nebular spectrum of SN 1987A do not have flat tops; indeed, they are all very similar in shape. That fact proves that the different element groups are not radially stratified, but are present in similar proportions throughout the line-emitting part of the envelope.

$\dagger$ Note that the line-emitting region is not the entire envelope, but only the inner, more slowly moving part of the envelope where the gas has greater density and where most of the gamma ray energy is deposited 


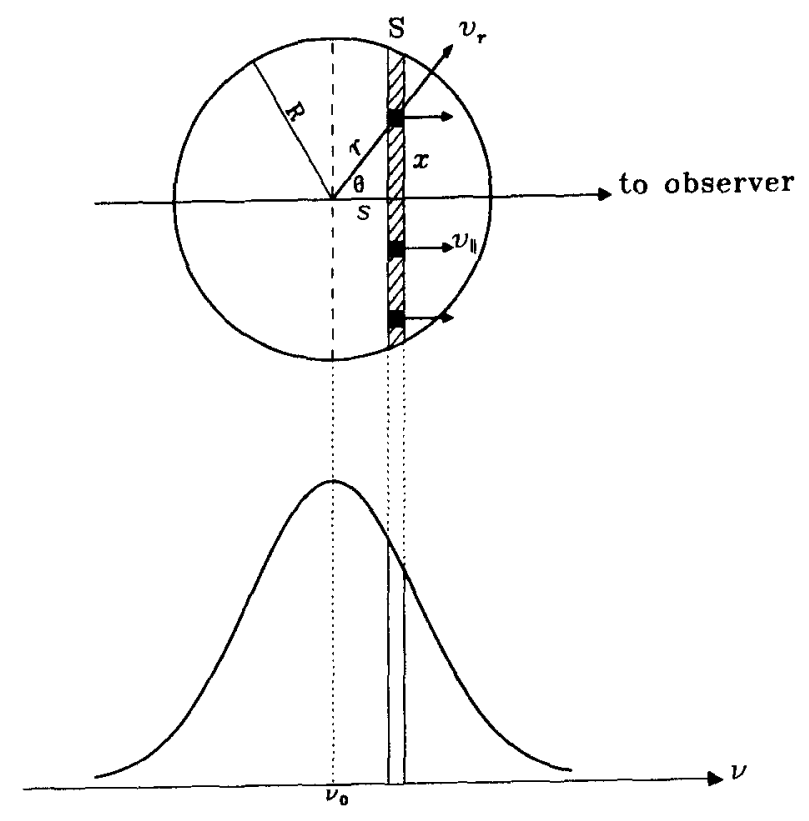

Figure 1. Line profile formation in a supernova envelope. The line emission from element $A$ at a given Doppler shift (lower panel) is confined to the intersection of the thin planar section (hatched) with the clumps (solid) containing element $A$ (upper panel).

\subsection{Line Optical Depth and the Sobolev Approximation}

Now we address the issue of line transfer. As we know, this can quickly become a nasty technical problem in a stellar atmosphere. In contrast, line transfer is easy to describe in a supernova envelope because of the hypersonic expansion. Generally, the optical depth is given by an expression of the form $\tau_{l u}=n_{a} \sigma_{l u}(\nu) \delta z$, where $n_{l}$ is the atomic density of absorbers in the lower $(l)$ state, $\sigma_{l u}(\nu)$ is the photon resonant scattering cross section, and $\delta z$ is the path length. Now, let us assume (to keep the algebra simple) that the normalized line profile function is a rectangle, with height $\phi(\delta \nu)=(\delta \nu)^{-1}$ and width $\delta \nu$. Then, a photon will remain in resonance with the line over a path length

$$
\delta z=c t \frac{\delta \nu}{\nu_{l u}} .
$$

But we can write $\sigma_{l u}(\nu)=\left(\pi e^{2} / m_{e} c\right) f_{l u} \phi(\delta \nu)$, where $f_{l u}$ is the absorption oscillator strength for the transition from the lower to upper $(u)$ level. Putting this all together, we derive an expression for the Sobolev optical depth of an absorption line in an expanding envelope:

$$
\tau_{l u}=\frac{\pi e^{2}}{m_{e} c} f_{l u} \lambda_{u l} t n_{l}\left[1-\frac{g_{l} n_{u}}{g_{u} n_{l}}\right]=\frac{c^{3} t g_{u} A_{u l} n_{l}}{8 \pi \nu^{3} g_{l}}\left[1-\frac{g_{l} n_{u}}{g_{u} n_{l}}\right],
$$

where we have included the term in square brackets to account for induced emission by a density, $n_{u}$, of atoms in the upper state, and the $g^{\prime} s$ are the statistical weights of the respective states. Note that for a freely expanding envelope, $n_{l} \propto t^{-3}$, so that $\tau_{l u} \propto t^{-2}$.

Now, the "Sobolev escape probability" that a resonance photon emitted by a downward 
transition will escape without further scattering is given by

$$
P_{e s c}=\int_{0}^{1} d \zeta \exp \left(-\zeta \tau_{l u}\right)=\tau_{l u}^{-1}\left[1-\exp \left(-\tau_{l u}\right)\right]
$$

and the line emissivity per unit volume is given by

$$
j_{u l}(\nu)=\frac{1}{4 \pi} n_{u} A_{u l} h \nu_{u l} \phi(\delta \nu) P_{e s c}\left(\nu_{u l}\right)
$$

where $A_{u l}$ is the spontaneous decay rate of the upper state. For a freely expanding supernova envelope the equation of transfer has the simple solution

$$
I_{u l}(\nu)=j_{u l}(\nu) \delta z=\frac{h c t}{4 \pi} n_{u} A_{u l} P_{e s c}\left(\nu_{u l}\right)
$$

Note that in the optically thick limit, $\tau_{l u} \gg 1, I_{u l}(\nu) \rightarrow B_{\nu}(T)$, as one would expect.

Although we have used the artifice of a rectangular line profile function to derive equations (2.2) and (2.5), the results are valid in general provided that the expansion Mach number is high.

The Sobolev opacity has the curious property that one can see line emission from the entire volume of the envelope even though the emission line may have high optical depth at every velocity. An opaque surface resonant at $\nu_{1}$ does not obscure the line emission from a surface deeper within the envelope resonant at $\nu_{2}\left\langle\nu_{1}-\delta \nu\right.$.

Consider now the total observed line flux, which is given by

$$
F_{u l}=\frac{1}{D^{2}} \int d \nu A(z) I_{u l}(\nu)
$$

where $D$ is the distance to the source and $A(z)$ is the cross-sectional area of the lineemitting gas at the plane corresponding to the frequency $\nu$ (see Figure 1).

Equation (2.6) has two important limits. In the optically thin limit, $\tau_{l u} \ll 1, P_{e s c} \rightarrow 1$ and we find

$$
F_{u l}=\frac{A_{u l} h \nu}{4 \pi D^{2}} \int d z A(z) n_{u}(z)=\frac{A_{u l} h \nu}{4 \pi D^{2}} N_{u},
$$

where $N_{u}$ is the total number of excited atoms in the emitting volume. If the atomic level populations are in LTE, we may write

$$
N_{u}=\frac{M_{A}}{m_{A}} \frac{g_{u}}{G(T)} \exp \left(-E_{u} / k T\right)
$$

where $M_{A}$ is the total mass of atoms, $m_{A}$ is their atomic mass, $E_{u}$ is their excitation energy, and $G(T)$ is the partition function. Note that equation (2.7) gives the result $F_{u l} \propto$ constant for constant $T$. Therefore, a decreasing line flux implies that the excitation temperature is decreasing.

In the optically thick limit, $\tau_{l u} \gg 1, P_{e s c} \rightarrow \tau_{l u}^{-1}$ and we find

$$
F_{u l}=\frac{\nu}{c t D^{2}} B_{\nu}(T) \int d z A(z)=\frac{\nu V_{A}}{c t D^{2}} B_{\nu}(T)
$$

where $V_{A}$ is the net volume occupied by atoms of element $A$ (the hatched volume in Figure 1). Since $V_{A} \propto t^{3}$ for free expansion, we find that $F_{u l} \propto t^{2}$ for constant $T$. The fact that the luminosities of several emission lines from SN 1987A actually do increase approximately as $t^{2}$ for $t \lesssim 200$ days is evidence that the lines are optically thick and that the temperature is fairly constant.

Note the difference between the optically thin limit (eq. 2.7) and the optically thick limit (eq. 2.9): in the former, the line flux is proportional to the mass of the emitting gas times a known function of temperature; whereas in the latter, the line flux is proportional 
to the volume occupied by the emitting gas times the Planck function. Therefore, if we can observe emission lines from two different excited levels of the same ion, we can infer the gas temperature from the ratio of the line strengths. Then, given the temperature, we can infer from the line fluxes either the net mass of the ion in question if the lines are optically thin or the volume occupied by that ion if the lines are optically thick.

The full width at zero intensity of the line profiles, $\Delta \lambda$, gives a measure of the total volume in which the emitting gas is contained (the volume of the sphere of radius $r_{m}=$ $v_{m} t$ in Figure 1),

$$
V_{E}=\frac{4 \pi}{3}\left(v_{m} t\right)^{3}=\frac{4 \pi}{3}\left[\frac{\Delta \lambda}{2 \lambda} c t\right]^{3},
$$

from which we may define the filling factor, $f_{A}=V_{A} / V_{E}$. In deriving equations (2.7), and (2.9), we have assumed that the temperature and density of the emitting gas are spatially uniform. Of course, one may wish to consider more complicated distributions of density and temperature in making detailed models for supernova spectra, in which case one can simply calculate the integral in equation (2.6) directly.

In the following, we will illustrate how the simple formalism described above can be used to infer the physical conditions in the envelope of SN 1987A from a few of the most prominent emission line systems in its nebular spectrum.

\section{Optically Thick Forbidden Lines: the [O I] $\lambda \lambda 6300$ Doublet}

The $[\mathrm{OI}] \lambda \lambda 6300$ doublet is one of the most prominent emission lines in the nebular spectrum of SN 1987A. The time evolution of the observed line ratio $R=F(6300) / F(6364)$ (actually, peak fluxes) is shown in Figure 2.

These observations provide a lovely example of how one can infer the density of a gas from Sobolev theory (Chugai 1990; Spyromilio \& Pinto 1991; Li \& McCray 1992). The two transitions, which couple the upper ${ }^{1} \mathrm{D}$ state to the lower ${ }^{3} \mathrm{P}$ state, have spontaneous decay rates related by $A(6300)=3 A(6364)$. Therefore, if the transitions were optically thin, the line ratio should be $R=3$, according to equation (2.7). But if both transitions were optically thick, then both lines should have identical intensity given by equation (2.9). In fact, we see the line ratio change from $R \approx 1$ at $t \approx 100$ days to $R \approx 2.5$ at $t \approx 500$ days (at which time the doublet is too weak to measure $R$ ). Clearly, we are seeing these emission lines make the transition from optically thick to optically thin.

From equations $(2.2,2.3,2.5$, and 2.6$)$, we derive the simple result:

$$
R=\frac{1-\exp [-\tau(6300)]}{1-\exp [-\tau(6364)]}=\frac{1-\exp \left[-3\left(t_{1} / t\right)^{2}\right]}{1-\exp \left[-\left(t_{1} / t\right)^{2}\right]}
$$

where, in deriving the second expression, we have defined $t_{1}$ as the time when $\tau(6364)=1$ and we have used the facts that $\tau \propto t^{-2}$ and $\tau(6300)=3 \tau(6364)$. The curve in Figure 2 represents a fit of equation (3.11) to the data; it gives $t_{1}=251$ days. Then, we may set $\tau(6364)=1$ in equation $(2.2)$ to derive $n_{l}\left(t_{1}\right)=n_{O I}\left(t_{1}\right) / 3$. The result is $n_{O I}(t)=4.0 \times 10^{9}\left(t / t_{1}\right)^{-3} \mathrm{~cm}^{-3}$.

If the spectrum of SN 1987A displayed other OI emission lines, we would be able to infer the mass, $M_{O}$, and temperature evolution, $T_{O}(t)$, of the OI-emitting gas from the emission line fluxes, as described in $\S 2$. Unfortunately, this is not the case. Therefore, $\mathrm{Li}$ $\&$ McCray (1992) simply estimated $M_{O} \approx 1.3 M_{\odot}$ in accord with supernova nucleosynthesis models (e.g., Nomoto et al 1989) in order to infer $T_{O}(t)$ from the observations. They find that $T_{O}(t)$ decreases from $T_{O}(200 d) \approx 4000 \mathrm{~K}$ to $T_{O}(500 d) \approx 3000 \mathrm{~K}$.

This result raises a very important general point: thermally excited optical emission 


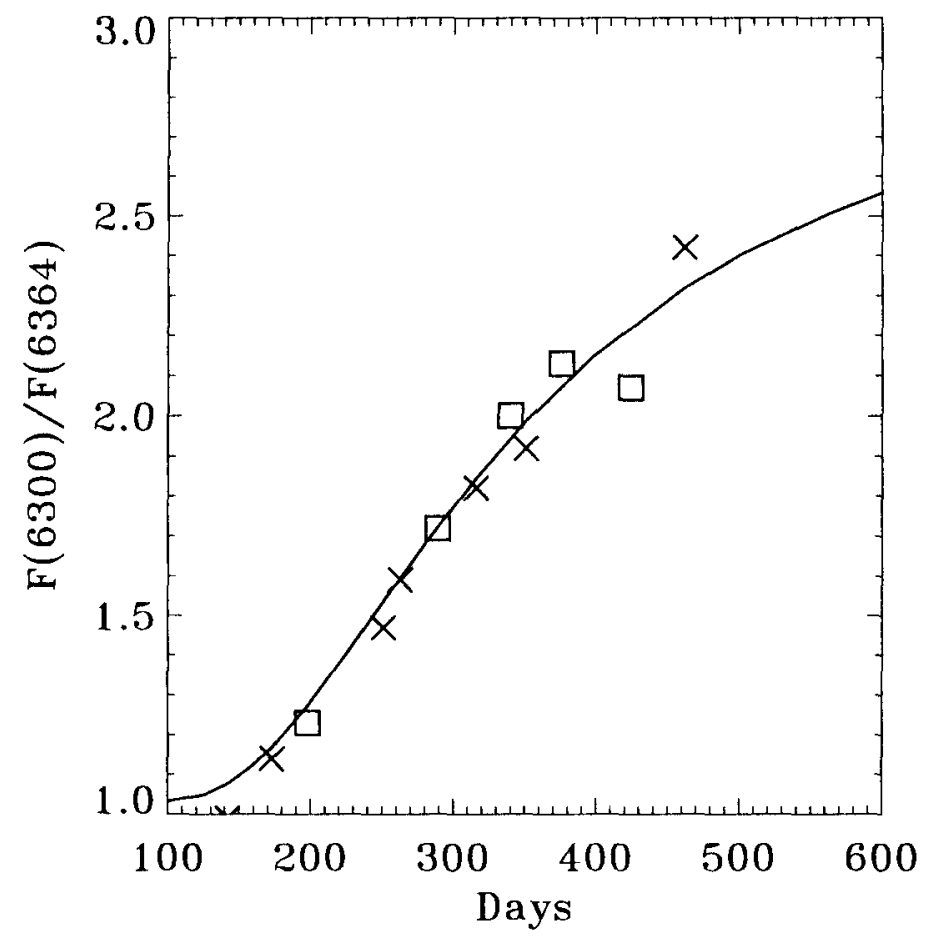

FIgURE 2. Variation with time of the ratio of peak fluxes $R=F(6300) / F(6364)$ of the [O I] $\lambda \lambda 6300$ doublet. Data are from Spyromilio \& Pinto (1991) (crosses) and Phillips \& Williams (1991) (stars). The solid curve is a fit to equation (3.11).

lines are poor diagnostics of supernova nucleosynthesis yields. The fluxes of optical emission lines are far more sensitive to temperature than to mass. For example, equations (2.6) and (2.8) give $F(6300) \propto M_{O} \exp \left(-22,800 / T_{O}\right)$. At $T \approx 3000 \mathrm{~K}$, a $10 \%$ increase in $T_{O}(t)$ will give the same increase in $F(6300)$ as a factor of 2 increase in $M_{O}$. By the same token, we can infer gas temperatures fairly accurately from the fluxes of optical emission lines even if our estimate of the emitting mass is very uncertain. But remember: the observed optical emission line fluxes are strongly biased by the hottest gas, which can easily obscure emission from a greater mass of cooler gas.

There is a way, however, to infer $M_{O}$ from the [OI] $\lambda \lambda 6300$ emission lines. As Fransson, Houck, \& Kozma (1995) point out, the excitation of these lines at late times $(t \gtrsim 750$ days) is dominated by nonthermal electrons produced by gamma ray energy deposition rather than by thermal electrons. Then, the emission line luminosity is proportional to the fraction of the gamma ray luminosity that is absorbed by the OI, which is in turn proportional to $M_{O} / M e n v$, since the gamma rays illuminate the entire envelope fairly uniformly at late times. From such considerations, Fransson et al infer $M_{O} \approx 1.6 M_{\odot}$, with fairly large uncertainty.

\section{Optically Thick Balmer Series: Case C Recombination}

The rich spectrum of hydrogen recombination lines from SN 1987A provides a fascinating example of the effects of line transfer (Chugai 1987; Xu et al 1992). Emission lines of $\mathrm{H} \alpha$ and several infrared lines of the Paschen, Brackett, Pfund, Humphreys series are prominent (see McCray 1993, Fig. 6). For $t \lesssim 2$ years, however, $\mathrm{H} \beta$ and the higher 
Balmer lines are absent because the envelope is optically thick in the Balmer series. When a recombination cascade results in the emission of an $\mathrm{H} \beta, \mathrm{H} \gamma$, or $\mathrm{H} \delta$ photon, these photons are resonantly absorbed by a nearby hydrogen atom in the $\mathrm{H}^{*}(n=2)$ state. This process of emission and resonance absorption repeats a few times until the Balmer line is "split," i.e. $\mathrm{H} \beta \rightarrow \mathrm{H} \alpha+\mathrm{P} \alpha, \mathrm{H} \gamma \rightarrow \mathrm{H} \alpha+\mathrm{P} \beta$, and so forth. $\mathrm{H} \alpha$, on the other hand, cannot split and so must continue to rattle around until it ultimately escapes. This situation, in which all the higher Balmer lines are split into $\mathrm{H} \alpha$ plus infrared recombination lines, may be called "Case C" recombination by analogy to the standard "Case B," for planetary nebulae and HII regions, in which the Lyman series is optically thick and $\mathrm{Ly} \beta \rightarrow \mathrm{Ly} \alpha+\mathrm{H} \alpha$, etc.

It is easy to understand these results using the formalism described in $\S 2$. First, consider the optical depth in Ly $\alpha$. The density, $n_{H}$, of $\mathrm{HI}$ atoms is given by

$$
n_{H}=\frac{0.7 M_{H}}{m_{H} f_{H} V_{E}} \approx 8 \times 10^{8} f_{H}^{-1} \frac{M_{H}}{M_{\odot}} t_{y}^{-3},
$$

where we have assumed that the line-emitting hydrogen (with total mass $M_{H}$ including $30 \%$ helium) occupies a filling factor $f_{H}$ of a sphere expanding with radial velocity $2000 \mathrm{~km} \mathrm{~s}^{-1}$, and $t_{y}$ is the time since explosion in years. Then, from eq. (2.2) we derive $\tau(\mathrm{Ly} \alpha) \sim 3 \times 10^{9} f_{H}^{-1}\left(M_{H} / M_{\odot}\right) t_{y}^{-2}$. Accordingly, if a hydrogen atom is excited to the $n=2$ level, the effective rate for decay by Ly $\alpha$ emission is $A_{21} / \tau(\operatorname{Ly} \alpha) \sim$ $0.14 \mathrm{~s}^{-1} f_{H}\left(M_{H} / M_{\odot}\right)^{-1} t_{y}^{2}$. Thus, for several years, the $n=2$ level is effectively metastable and will decay instead by two-photon continuum emission, at a rate $A_{2 \gamma}=2.05 \mathrm{~s}^{-1}$.

We can calculate the optical depths in the Balmer series from equation eq. (2.2) if we know the density, $n_{2}$, of $\mathrm{HI}$ atoms in the $n=2$ level. We can estimate $n_{2}$ by equating excitations and ionizations of $\mathrm{HI}$ (all of which populate the $n=2$ level) due to gamma ray energy deposition (see Fransson et al 1995) to 2-photon decays. Following Xu et al (1992), we estimate that the Balmer continuum optical depth is given by

$$
\tau_{B C} \approx 8 \times 10^{3} t_{y}^{-2} \exp \left(-t_{y} / 0.3\right)\left[1-f_{\gamma}\right]
$$

where $f_{\gamma}$ is the fraction of the gamma ray luminosity that escapes the line-emitting region (see McCray 1993, Figure 2). According to eq. (4.13), the Balmer continuum should remain optically thick for about two years. If so, the ionization rate of $\mathrm{HI}$ by gamma rays is enhanced by a factor $5-10$ because every ionization of $\mathrm{HI}$ from the ground state is accompanied by several photoionizations of metastable $\mathrm{HI}(n=2)$ due to Balmer continuum photons produced by 2-photon decays (Fransson et al 1995). This is clearly happening in SN 1987A; in fact, as Xu et al (1992) show, one cannot account for the strength of the observed recombination lines without this enhancement. But shortly after two years, the Balmer continuum and $\mathrm{H} \beta$ become optically thin, the enhancement vanishes, and the recombination spectrum changes to the more familiar Case $B$.

\section{A Cautionary Tale: the Ca II Infrared Lines}

The strongest emission features in the near infrared spectrum of SN 1987A are the [Ca II] $\lambda \lambda 7300$ and the Ca II $\lambda \lambda 8600$ infrared triplet. Li \& McCray (1993) have interpreted these observations, following the pioneering work of Kirshner \& Kwan (1975), and their results provide an important lesson about interpreting supernova spectra.

The relevant transitions and energy levels are shown in Figure 3. The $[\mathrm{CaII}] \lambda \lambda 7300$ doublet is unresolved. Since the transitions are highly forbidden, the upper $3 d^{2} D$ states are in LTE with the ground state. In contrast, the CaII $\lambda \lambda 8600$ transitions are allowed, and the $\lambda 8662$ line can be distinguished from the $\lambda \lambda 8498,8542$ blend. Therefore, the 


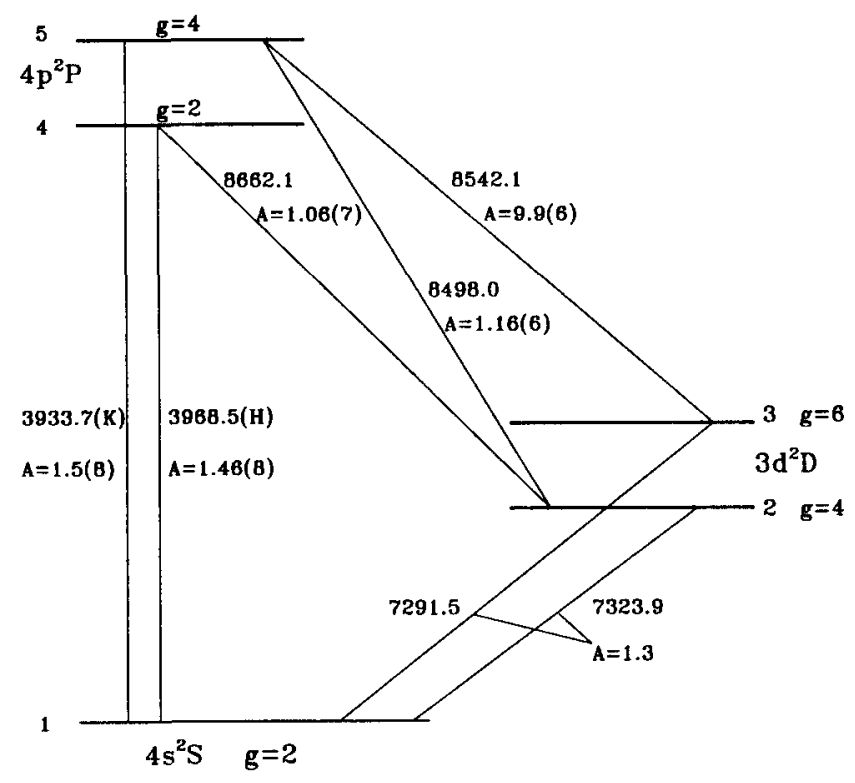

FIgure 3. Grotrian diagram of CaII levels. Statistical weights and Einstein $A$ values $\left(\mathrm{s}^{-1}\right)$ are indicated. The numbers in parentheses are the powers of 10 .

upper $4 p^{2} P$ states are not in LTE, and in fact their populations are sensitive to temperature, electron density, and the radiation field in the neighborhood of Ca II $\lambda \lambda 3950$.

Li \& McCray (1993) analyzed these observations with a 5-level atom model that includes all radiative and electron-impact transitions. They fit the data with parameters representing $M_{C a}$, the calcium mass, $f_{C a}$, the calcium filling factor, $T_{C a}(t)$ the temperature of the CaII-emitting gas, $n_{e}(t)$, the electron density, and a $\zeta_{H, K}$, the rate of pumping of the $4 p^{2} P$ levels due to absorption of ultraviolet photons by the CaII $\lambda \lambda 3950$ $(\mathrm{H}, \mathrm{K})$ doublet.

Their conclusions are remarkable. First, they could not fit the observations with a typical nucleosynthesis yield of calcium, $M_{C a} \sim(3-10) \times 10^{-3} M_{\odot}$ (Woosley 1991; Nomoto 1991) and a relatively small filling factor. In fact, the good fits have a relatively large filling factor, $f_{C a} \gtrsim 0.1$ and $M_{C a} \sim(2-5) \times 10^{-4} M_{\odot}$. Moreover, the Ca II infrared emission lines provide an excellent thermometer of the calcium-emitting gas, and imply temperatures ranging from, $T_{C a}(200 d) \approx 6300 \mathrm{~K}$ to $T_{C a}(500 d) \approx 3800 \mathrm{~K}$ - substantially higher than the temperatures $T_{O}(t)$ of the $[\mathrm{O} \mathrm{I}] \lambda \lambda 6300$ emitting gas at the same times.

From this analysis, Li \& McCray conclude: the observed Ca II lines are emitted, not by newly-synthesized calcium, but by primordial calcium in the hydrogen-and helium-rich parts of the envelope.

Does this conclusion preclude the nucleosynthesis yields of calcium predicted by Woosley (1991) and Nomoto (1991)? Not at all! Since the fluxes of the CaII near-infrared emission lines are very sensitive to temperature, the emission from newly-synthesized calcium would be masked by the emission from a much lower mass of primordial calcium mixed with hydrogen and helium if the newly-synthesized calcium has substantially lower temperature.

Indeed, a simple argument shows that this must be the case. Since the gamma rays have relatively long mean-free paths, the fraction of the gamma ray luminosity that can be absorbed by clumps of newly-synthesized calcium and other $Z \approx 20$ elements must be 
proportional to the mass fraction of the emitting region occupied by such material. That fraction must be an upper limit to the fraction of the supernova luminosity that can be emitted by newly-synthesized calcium; but in fact, the net luminosity of the observed $\mathrm{Ca}$ II lines exceed that limit. Indeed, the only way to account for this luminosity is to assume that the emitting CaII is mixed microscopically with a major fraction of the mass of the emitting region. Since the emitting mass must be $z 5 M_{\odot}$ in order to absorb the gamma rays (McCray 1993), it can only be hydrogen and helium. A relatively large fraction of the absorbed gamma ray luminosity emerges in the Ca II infrared lines, not because $\mathrm{Ca}$ II is particularly abundant, but because it is one of the most effective radiative cooling agents in primordial gas at temperatures characteristic of the envelope.

Li \& McCray (1993) noticed one more important point: for $t z 350$ days, the emission in the $\mathrm{Ca} I I \lambda \lambda 8600$ lines must be driven by ultraviolet pumping in the CaII $\lambda \lambda 3950$ lines rather than by electron impact excitation.

\section{Clues to Explosion Dynamics: the Iron, Cobalt and Nickel Lines}

Light curves of more than a dozen emission lines from neutral and once-ionized Fe, Co, and $\mathrm{Ni}$ have been observed in the infrared spectrum of SN 1987A. There is no doubt that these lines are emitted by newly-synthesized material. Indeed, one can directly observe the radioactive decays of ${ }^{56} \mathrm{Co} \rightarrow{ }^{56} \mathrm{Fe}$ (mean lifetime $t_{56}=111.3 d$ ) and ${ }^{57} \mathrm{Co} \rightarrow{ }^{57} \mathrm{Fe}$ $\left(t_{57}=391 d\right)$ and infer the initial production ratio of these isotopes from observations of the evolution of the ratio [Co II] $\lambda 1.547 \mu \mathrm{m} /[\mathrm{Fe} \mathrm{II}] \lambda 1.533 \mu \mathrm{m}$ (Varani et al 1990).

Since we can observe the entire bolometric luminosity of SN 1987A, and we know that it results from the radioactive decays of ${ }^{56} \mathrm{Co}$ and ${ }^{57} \mathrm{Co}$, we know the masses of the respective isotopes of $\mathrm{Fe}, \mathrm{Co}$, and $\mathrm{Ni}$ as functions of time. For example, $M\left({ }^{56} \mathrm{Co}\right)=$ $\left(0.07 M_{\odot}\right) \exp (-t / 111.3 d)$. Therefore, if we assume that the gas responsible for the $\mathrm{Fe} / \mathrm{Co} / \mathrm{Ni}$ emission line system is distributed throughout the emitting region in clumps, each having the same density and temperature, the development of this system should be characterized fully by the net filling factor of these clumps, $f_{F e}$, and the evolution of temperature, $T_{F e}(t)$, and ionization levels, $x_{F e}=n(F e I I) / n(F e)$, etc.

$\mathrm{Li}, \mathrm{McCray}, \&$ Sunyaev (1993) have developed a theory to account for the evolution of the $\mathrm{Fe} / \mathrm{Co} / \mathrm{Ni}$ emission line system. Their most important conclusion is that the filling factor, $f_{F e} z 0.25$. This conclusion follows directly from the theory outlined in $\S 2$. If we replace $B_{\nu}(T)$ by the Rayleigh-Jeans limit, we can write equation (2.9) as an upper limit that is valid in general:

$$
F_{u l}<f_{A} \frac{4 \pi v_{m}^{3} t^{2}}{3 D^{2}} \frac{2 k T}{\lambda^{3}}
$$

Consider, for example, the [CoII] $10.52 \mu \mathrm{m}$ emission line, which reaches maximum brightness $F(10.52) \approx 3 \times 10^{-10}$ ergs $\mathrm{cm}^{-2} \mathrm{~s}^{-1}$ at $t \approx 250$ days. With these values, $v_{m}=$ $2500 \mathrm{~km} \mathrm{~s}^{-1}$, and $D=50 \mathrm{kpc}$, equation (6.14) implies that $f_{F e} T_{3}>1.0$, where $T_{3}=$ $T_{F e} /\left(10^{3} \mathrm{~K}\right)$. Now, since we can infer from $\mathrm{Fe} / \mathrm{Co} / \mathrm{Ni}$ line ratios that $T_{\mathrm{Fe}} \approx 4000 \mathrm{~K}$, equation (6.14) implies that $f_{F e} \gtrsim 0.25$. [This result was anticipated by Chugai (1988), Moseley et al (1989), and Haas et al (1990)].

This result is remarkable because we know that the net mass, $M_{F e} \approx 0.07 M_{\odot}$, of the $\mathrm{Fe} / \mathrm{Co} / \mathrm{Ni}$ is only about $1 \%$ of the total mass, $M_{e m} \sim 5-10 M_{\odot}$, of the emitting gas, which must be mostly hydrogen and helium. (Such a mass is necessary to absorb the gamma rays.) It follows that the emitting region has a foamy texture, consisting of low-density bubbles of $\mathrm{Fe} / \mathrm{Co} / \mathrm{Ni}$ gas in a substrate of higher density hydrogen and helium.

Why should this be so? The answer was anticipated by Woosley (1988), who pointed 
out that the decay of ${ }^{56} \mathrm{Ni}$ during the first few weeks might deposit enough thermal energy to cause the $\mathrm{Fe} / \mathrm{Co} / \mathrm{Ni}$ gas to expand relative to its substrate, forming a "nickel bubble." But there is a problem: the net radioactive energy released by the decay of $0.07 M_{\odot}$ of ${ }^{56} \mathrm{Ni}, \approx 4 \times 10^{48} \mathrm{ergs}$, is much less than the kinetic energy, $\approx 2 \times 10^{50} \mathrm{ergs}$, of an expanding uniform sphere of mass $M \approx 5 M_{\odot}$ and surface velocity $v_{m}=2500 \mathrm{~km} \mathrm{~s}^{-1}$. How can the $\mathrm{Fe} / \mathrm{Co} / \mathrm{Ni}$ clumps push aside such a large fraction of the volume of such a sphere with so little energy? The answer is that there must be many $(N \gtrsim 60)$ small clumps originally distributed throughout the substrate (presumably as a result of hydrodynamic instabilities during the explosion). Then, each clump can expand by a factor $\sim 10^{2}$ without changing the original velocity of the substrate by more than, say, $10 \%$. I.e., many small bubbles dispersed through the substrate can push aside a large fraction of the volume much more efficiently than one large bubble.

Starting with these elementary considerations, Li et al (1993) develop a fairly complete theory for the light curves of all the $\mathrm{Fe} / \mathrm{Co} / \mathrm{Ni}$ emission lines from SN 1987A. They calculate $T_{\mathrm{Fe}}(t)$ by equating the radioactive luminosity deposited in the $\mathrm{Fe} / \mathrm{Co} / \mathrm{Ni}$ gas to the net luminosity of all the emission lines, and $x_{F e}(t), x_{C o}(t)$, and $x_{N i}(t)$ by equating ionization to recombination. From that they can calculate the light curves of all the lines $a b$ initio. The theory has essentially no free parameters except the filling factor, $f_{F e}$, which is determined by observations. The observed light curves of most of the emission lines agree very well with the theoretical light curves.

There is an important exception, however. The observed fluxes of near infrared lines, such as [Fe II] $\lambda 7155$, agree fairly well with the theoretical light curves for $t \lesssim 400$ days, but are much greater than the theoretical results thereafter. (The light curves of the longer wavelength lines agree with the theory for all times.) Evidently, some small fraction of the $\mathrm{Fe} / \mathrm{Co} / \mathrm{Ni}$ atoms are hotter than the rest, and dominate the light curves of the shorter wavelength lines at late times. $\mathrm{Li}$ et al (1993) suggest that these hotter atoms reside on the surfaces of the $\mathrm{Fe} / \mathrm{Co} / \mathrm{Ni}$ bubbles, and are heated by ionizing ultraviolet radiation from the hydrogen/helium substrate. They do not prove this conjecture, however.

\section{The Unfinished Agenda}

Clearly, we have made substantial progress toward a theory of the spectrum of SN 1987A. We have a fairly complete theory for the $\mathrm{Fe} / \mathrm{Co} / \mathrm{Ni}$ lines. We also have semi-empirical theories for the OI lines, the hydrogen recombination lines, and the Ca II lines; but these models are incomplete because the gas temperature is treated as a parameter of the theory rather than calculated by equating heating with cooling.

To do so, we must identify correctly the main radiative cooling agents of each chemically distinct constituent of the envelope. Here, the CaII lines teach us an important lesson: trace elements can be important, even dominant, cooling agents. Clearly, we should be guided by observations of the emission line spectrum in order not to overlook important cooling agents. For example, emission bands of $\mathrm{CO}$ and $\mathrm{SiO}$ are prominent in the infrared spectrum and must play an important role in cooling the oxygen-rich gas.

We have also learned that photoionization and photoexcitation by ultraviolet radiation play important roles in determining the hydrogen recombination line spectrum, the Ca II infrared lines, and the $\mathrm{Fe} / \mathrm{Co} / \mathrm{Ni}$ emission lines at late times. To calculate these processes from first principles, we need a theory to calculate the flux density of ultraviolet radiation within the envelope of SN 1987A. This radiation field is produced by non-thermal excitation resulting from gamma ray illumination and absorbed by resonance scattering by trace elements, dust, and other continuum processes. 
Finally, although we have made progress toward modeling the brightest emission lines in the nebular spectrum of SN 1987A, we have not accounted for many of the weaker features. Most significantly, we have no theory to explain the quasi-continuum (color temperature $T_{c} \sim 5000 \mathrm{~K}$ ) that underlies the optical emission line spectrum (Wooden $e t$ al 1993). Perhaps it is fluorescence following resonance absorption of ultraviolet radiation within the envelope.

\section{REFERENCES}

Chugai, N. N. 1987, Soviet Astron. Lett., 13, 282.

Chugai, N. N. 1988, Astron. Tsirk., 1533, 7.

Chugai, N. N. 1990, Soviet Astron. 34, 96.

Colgan, S. W. J., \& Hollenbach, D. J. 1988, ApJL, 329, L25.

Fransson, C., \& Chevalier, R. A. 1987. ApJL, 322, L15.

Fransson, C. Houck, J. \& Kozma, C. 1995, these proceedings.

Haas, M. R., Colgan, S. W. J., Erikson, E. F., Lord, S. D., Burton, M. G., \& Hollenbach, D. J. 1990, ApJ, 360, 257.

Kirshner, R., \& Kwan, J., 1975, ApJ, 197, 415.

Li, H.-W., \& McCray, R. 1992, ApJ, 387, 309.

Li, H.-W., \& McCray, R. 1993, ApJ, 405, 730.

Li, H.-W., McCray, R., \& Sunyeav, R. A. 1993, ApJ, 419, 824.

Luo, D., McCray, R., \& Slavin, J. 1994, ApJ, 430, 264.

Masai, K., \& Nomoto, K. 1994, ApJ, 424, 924.

McCray, R. 1993, Ann. Rev. Astr. \& Ap., 31, 175.

Moseley, S. H., Dwek, E., Glaccum, W., Graham, J. R., Loewenstein, R. F., \& Silverberg, R. F. 1989, ApJ, 347, 119.

Nomoto, K., Shigeyama, T., Kumagai, S., \& Yamaoka, H. 1991, in Supernovae, ed. S.E. Woosley (New York: Springer-Verlag), p. 176.

Osterbrock, D. E. 1989, Astrophysics of Gaseous Nebulae and Active Galactic Nuclei (Mill Valley, CA: University Science Books).

Phillips, M. M., \& Williams, R. 1991, in Supernovae, ed. S. E. Woosley (New York: SpringerVerlag), p. 36.

Spyromilio, J., \& Pinto, P. A. 1991, in SN1987A and Other Supernovae, ed. I. J. Danziger, K. Kjär. ESO/EIPC Workshop on Supernovae. Munich: ESO (1991), p. 423.

Suzuki, T., Shigeyama, T., and Nomoto, K. 1993, A\&A, 275, 883.

Swartz, D. A., Harkness, R. P., \& Wheeler, J. C. 1987, Nature 337, 439

Varani, G.-F., Meikle, W. P. S., Spyromilio, J., \& Allen, D. A. 1990, MNRAS, 245, 570.

Wheeler, J. C. \& Filippenko, A. V. 1995, these proceedings.

Wooden, D. H., et al 1993, ApJS, 88, 477.

Woosley, S. E. 1988, ApJ, 330, 218.

Woosley, S. E. 1991, in Supernovae, ed. S. E. Woosley (New York: Springer-Verlag), p. 202.

Xu, Y., McCray, R., Oliva, E., \& Randich, S. 1992, ApJ, 386, 181. 